NASA Technical Memorandum 102539

ICOMP-90-10

\title{
High Accuracy Solutions of Incompressible Navier-Stokes Equations
}

Murli M. Gupta

The George Washington University

Washington, D.C.

and Institute for Computational Mechanics in Propulsion Lewis Research Center

Cleveland, Ohio

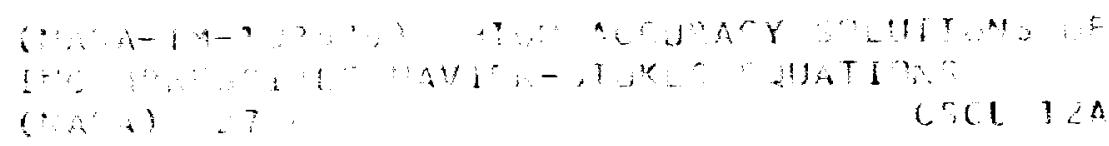

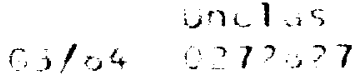

March 1990

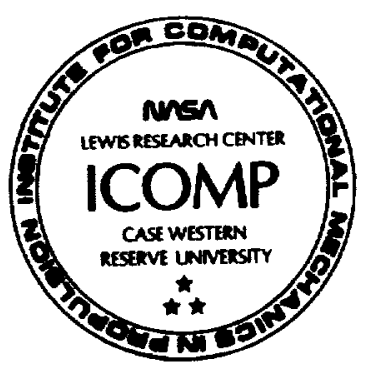



High Accuracy Solutions of Incompressible Navier-stokes
Equations

\author{
Murli M. Gupta \\ Department of Mathematics \\ The George Washington University \\ Washington, D.C. 20052 \\ and Institute for Computational Mechanics in Propulsion \\ NASA Lewis Research Center \\ Cleveland, Ohio 44135
}

\title{
SUMMARY
}

In recent years we have developed high accuracy finite difference approximations for partial differential equations of elliptic type, with particular emphasis on the convection-diffusion equation. These approximations are of compact type, have a local truncation error of fourth order, and allow the use of standard iterative schemes to solve the resulting systems of algebraic equations. In this paper, we extend these high accuracy approximations to the solution of Navier- Stokes equations. Solutions are obtained for the model problem of driven cavity and are compared with solutions obtained using other approximations and those obtained by other authors. It is discovered that the high order approximations do indeed produce high accuracy solutions and have a potential for use in solving important problems of viscous fluid flows.

*Work funded by Space Act Agreement C-99066-G. 
INTRODUCTION

The basic model for the fluid dynamics phenomena representing two and three dimensional flows of an incompressible viscous fluid is given by the Navier- Stokes equations that represent the conservation of mass, momentum and energy. These equations are highly nonlinear and are very difficult to solve, especially when the approximate solutions are required to have a high accuracy. A related problem is that of obtaining highly accurate solutions of the convection- diffusion equation especially when convection is the dominating phenomena.

Prior to the last decade, many researchers examined a number of first and second order finite difference and finite element methods that were accurate and stable. In the area of finite difference methods, it was discovered that although central difference approximations were locally second order accurate they of ten suffered from computational instability and the resulting solutions exhibited nonphysical oscillations. The upwind difference approximations were computationally stable though only first order accurate and the resulting solutions exhibited the effects of artificial viscosity. The second order upwind methods were no better and the higher order finite difference methods of conventional type were computationally inefficient. 
An exception has been found in the high order finite difference schemes of compact type that are computationally efficient and stable and yield highly accurate numerical solutions at least for the linear and quasilinear partial differential equations. Simplest version of such compact schemes is given for the Poisson equation

$$
\partial^{2} u / \partial x^{2}+\partial^{2} u / \partial y^{2}=f(x, y)
$$

which can be discretized at a grid point $(x, y)$ by a nine point finite difference approximation:

$$
\begin{aligned}
4\left[u_{1}+u_{2}+u_{3}+u_{4}\right]+u_{5}+u_{6}+u_{7}+u_{8}-20 u_{0} \\
=h^{2}\left[f_{1}+f_{2}+f_{3}+f_{6}+8 f_{0}\right] / 2 .
\end{aligned}
$$

(See Figure 1 for the computational stencil). This approximation was named Mehrstellenverfahren by Collatz [3] (see also [10]). It has a local truncation error of order $\mathrm{h}^{4}$ and is an approximation of compact type as it involves only the eight nearest neighbors of the point $(x, y)$. This type of approximations have been obtained for other elliptic equations by many researchers: the Hodie schemes of Lynch and Rice [14] (see also [2]), the O.C.I. schemes of Berger et al.[1], and the SCHOS schemes of Gupta et al. $[12,13]$ all reduce to the above difference approximation when applied to the Poisson equation. Similar compact schemes of order $h$ " have also been obtained [15]. 
The compact schemes of Gupta et al. (called ScHos in the earlier papers) were applied to the convection-diffusion equations in particular and were found to yield high accuracy when applied to a large number of test problems including problems of convection dominated flows [12]. In this paper, we extend these finite difference schemes to the Navier-Stokes equations. As a test of this method, we solve the model problem of lid driven cavity for small to moderate values of the Reynolds number and compare our numerical solutions with the highly accurate benchmark solutions available in the literature. We $f$ ind that our method yields high accuracy even though we use a relatively coarse grid.

In the next section we describe the fourth order compact difference schemes for the convection-diffusion equation and for the Navier-Stokes equations. The model problem of the lid driven cavity is described in the following section that follows with detailed comparisons of our solutions with the existing solutions in the literature.

\section{FINITE DIFFERENCE APPROXIMATIONS}

Consider the following steady convection- diffusion equation:

$$
\partial^{2} u / \partial x^{2}+\partial^{2} u / \partial y^{2}+p(x, y) \partial u / \partial x+q(x, y) \partial u / \partial y=f(x, y)
$$


Finite difference approximations of eq.(1) at the grid point $(x, y)$ are obtained in terms of the function values of $u, p$, $q$, and $f$ at $(x, y)$ and its neighbours. Assuming a uniform grid in both $x$ and $y$ directions, we number the grid points $(x, y),(x+h, y),(x, y+h),(x-h, y),(x, y-h),(x+h, y+h)$, $(x-h, y+h),(x-h, y-h),(x+h, y-h)$ as $0,1,2,3,4,5,6,7,8$, respectively (see Figure 1 ). In writing the finite difference approximations a single subscript ' $j$ ' denotes the corresponding function value at the grid point numbered ' $j$ '.

The usual central difference approximation of eq.(1) at the point $(x, y)$ is given by

$$
\begin{aligned}
& {\left[u_{1}+u_{2}+u_{3}+u_{4}-4 u_{0}\right]} \\
& +p_{0} h\left(u_{1}-u_{3}\right) / 2+q_{0} h\left(u_{2}-u_{4} / 2=h^{2} f_{0} .\right.
\end{aligned}
$$

This approximation is obtained by replacing all derivatives in eq. (1) by central differences that have truncation errors of second order. A high accuracy approximation of eq.(1) at the grid point $(x, y)$ is given by

$$
\begin{aligned}
& c_{1} u_{1}+c_{2} u_{2}+c_{3} u_{3}+c_{4} u_{4}+c_{5} u_{5}+c_{6} u_{6}+c_{1} u_{7}+c_{8} u_{8}-c_{0} u_{0} \\
& =h^{2}\left[f_{1}+f_{2}+f_{3}+f_{4}+8 f_{0}\right] / 2+h^{3}\left[p_{0}\left(f_{1}-f_{3}\right)+q_{0}\left(f_{2}-f_{4}\right)\right] / 4,
\end{aligned}
$$

where,

$$
\begin{aligned}
c_{1}=4+ & h / 4\left(4 p_{0}+3 p_{1}+p_{2}-p_{3}+p_{4}\right) \\
& +h^{2} / 8\left[4 p_{0}^{2}+p_{0}\left(p_{1}-p_{3}\right)+q_{0}\left(p_{2}-p_{4}\right)\right], \\
c_{2}=4+ & h / 4\left(4 q_{0}+q_{1}+3 q_{2}+q_{3}-q_{4}\right) \\
& +h^{2} / 8\left[4 q_{0}^{2}+p_{0}\left(q_{1}-q_{3}\right)+q_{0}\left(q_{2}-q_{4}\right)\right],
\end{aligned}
$$




$$
\begin{aligned}
& c_{3}=4- h / 4\left(4 p_{0}-p_{1}+p_{2}+3 p_{3}+p_{4}\right) \\
&+h^{2} / 8\left[4 p_{0}^{2}-p_{0}\left(p_{1}-p_{3}\right)-q_{0}\left(p_{2}-p_{4}\right)\right], \\
& c_{4}=4- h / 4\left(4 q_{0}+q_{1}-q_{2}+q_{3}+3 q_{4}\right) \\
&+h^{2} / 8\left[4 q_{0}^{2}-p_{0}\left(q_{1}-q_{3}\right)-q_{0}\left(q_{2}-q_{4}\right)\right], \\
& c_{5}=1+ h / 2\left(p_{0}+q_{0}\right)+h / 8\left(q_{1}+p_{2}-q_{3}-p_{4}\right)+h^{2} / 4 p_{0} q_{0}, \\
& c_{6}=1- h / 2\left(p_{0}-q_{0}\right)-h / 8\left(q_{1}+p_{2}-q_{3}-p_{4}\right)-h^{2} / 4 p_{0} q_{0}, \\
& c_{7}=1-h / 2\left(p_{0}+q_{0}\right)+h / 8\left(q_{1}+p_{2}-q_{3}-p_{4}\right)+h^{2} / 4 p_{0} q_{0}, \\
& c_{8}=1+h / 2\left(p_{0}-q_{0}\right)-h / 8\left(q_{1}+p_{2}-q_{3}-p_{4}\right)-h^{2} / 4 p_{0} q_{0}, \\
& c_{0}=20+ h^{2}\left(p_{0}^{2}+q_{0}^{2}\right)+h\left(p_{1}-p_{3}\right)+h\left(q_{2}-q_{4}\right),
\end{aligned}
$$

This approximation is of compact type as it involves only the nine grid values of $u$ at the point $(x, y)$ and its eight nearest neighbors (see Figure 1). This approximation has a local truncation error of order $h^{4}$. Detailed derivation of this approximation is given by Gupta et al. [12]. (This approximation has recently been rediscovered by Dennis and Hudson [21].) Similar high order approximation for general second order elliptic equations are given in [13]. Other compact approximations of this type have been obtained for the Poisson equation [10], the Helmholtz equation [15], and the biharmonic equation [19]. Results of computations with a large number of test problems have been reported in the cited papers and in each case these compact schemes are found to yield highly accurate numerical solutions. Moreover, the accuracy improves rapidly, consistent with the local truncation error, as the mesh is refined. 
The Navier- Stokes equations representing the twodimensional steady flow of an incompressible viscous fluid are given in streamfunction-vorticity form as follows:

$$
\begin{aligned}
& \partial^{2} \psi / \partial x^{2}+\partial^{2} \psi / \partial y^{2}=-\zeta \\
& \partial^{2} \zeta / \partial x^{2}+\partial^{2} \zeta / \partial y^{2}-\operatorname{Re}(u \partial \zeta / \partial x+v \partial \zeta / \partial y)=0 \\
& u=\partial \psi / \partial y, \quad v=-\partial \psi / \partial x
\end{aligned}
$$

Here $\psi$ is the streamfunction, $\zeta$ the vorticity; $u, v$ are the velocities; $\operatorname{Re}$ is the nondimensional Reynolds number.

The streamfunction equation (5) is a special case of eq. (1). The fourth order compact approximation for this equation may be obtained by putting $u=\psi, f=-\zeta$ and $p(x, y)=0, q(x, y)=0$ in eq. $(3)$ :

$$
\begin{aligned}
4\left[\psi_{1}+\psi_{2}+\psi_{3}+\psi_{4}\right] & +\psi_{5}+\psi_{6}+\psi_{7}+\psi_{8}-20 \psi_{0} \\
= & -h^{2}\left[\zeta_{1}+\zeta_{2}+\zeta_{3}+\zeta_{4}+8 \zeta_{0}\right] / 2 .
\end{aligned}
$$

The vorticity equation (6) is also a special case of eq.(1) and the fourth order approximation in this case may be obtained by putting $u=\zeta, f=0$ and $p(x, y)=-\operatorname{Re} u(x, y)$, $q(x, y)=-\operatorname{Re} v(x, y)$ in eq. $(3),(4)$ :

$$
\sum_{i=1}^{8} c_{i} \zeta_{i}-c_{0} \zeta_{0}=0, \quad \text { where }
$$




$$
\begin{aligned}
& C_{1}=4-\operatorname{Reh} / 4\left(4 u_{0}+3 u_{1}+u_{2}-u_{3}+u_{4}\right) \\
& +(\operatorname{Reh})^{2} / 8\left[4 u_{0}^{2}+u_{0}\left(u_{1}-u_{3}\right)+v_{0}\left(u_{2}-u_{4}\right)\right] \text {, } \\
& C_{2}=4-\operatorname{Reh} / 4\left(4 v_{0}+v_{2}+3 v_{2}+v_{3}-v_{4}\right) \\
& +(\operatorname{Reh})^{2} / 8\left[4 v_{0}^{2}+u_{0}\left(v_{2}-v_{3}\right)+v_{0}\left(v_{2}-v_{4}\right)\right] \text {, } \\
& c_{3}=4+\operatorname{Reh} / 4\left(4 u_{0}-u_{1}+u_{2}+3 u_{3}+u_{4}\right) \\
& +(\operatorname{Reh})^{2} / 8\left[4 u_{0}^{2}-u_{0}\left(u_{1}-u_{3}\right)-v_{0}\left(u_{2}-u_{4}\right)\right] \text {, } \\
& C_{4}=4+\operatorname{Reh} / 4\left(4 v_{0}+v_{1}-v_{2}+v_{3}+3 v_{4}\right) \\
& +(\operatorname{Reh})^{2} / 8\left[4 v_{0}^{2}-u_{0}\left(v_{1}-v_{3}\right)-v_{0}\left(v_{2}-v_{4}\right)\right] \text {, } \\
& c_{5}=1-\operatorname{Reh} / 2\left(u_{0}+v_{0}\right)-\operatorname{Reh} / 8\left(v_{1}+u_{2}-v_{3}-u_{4}\right)+(\operatorname{Reh})^{2} u_{0} v_{0} / 4 \text {, } \\
& c_{6}=1+\operatorname{Reh} / 2\left(u_{0}-v_{0}\right)+\operatorname{Reh} / 8\left(v_{1}+u_{2}-v_{3}-u_{4}\right)-(\operatorname{Reh})^{2} u_{0} v_{0} / 4 \text {, } \\
& c_{7}=1+\operatorname{Reh} / 2\left(u_{0}+v_{0}\right)-\operatorname{Reh} / 8\left(v_{1}+u_{2}-v_{3}-u_{4}\right)+(\operatorname{Reh})^{2} u_{0} v_{0} / 4 \text {, } \\
& c_{s}=1-\operatorname{Reh} / 2\left(u_{0}-v_{0}\right)+\operatorname{Reh} / 8\left(v_{1}+u_{2}-v_{3}-u_{4}\right)-(\operatorname{Reh})^{2} u_{0} v_{0} / 4 \text {, } \\
& c_{0}=20+(\operatorname{Reh})^{2}\left(u_{0}^{2}+v_{0}^{2}\right)-\operatorname{Reh}\left(u_{1}-u_{3}\right)-\operatorname{Reh}\left(v_{2}-v_{4}\right) \text {. }
\end{aligned}
$$

The velocities $u, v$ at a grid point $(x, y)$ are calculated from the discrete approximations of eq.(7). The typical second order central difference approximations for the velocities are:

$$
u_{0}=\left(\psi_{2}-\psi_{4}\right) / 2 h, v_{0}=\left(\psi_{3}-\psi_{2}\right) / 2 \mathrm{~h}
$$

High order approximations for the velocities $u, v$ can also be defined. We [10] earlier derived some high accuracy compact approximations for the gradients of the solution of Poisson equations. As the streamfunction equation (5) is a Poisson equation in $\psi$, high accuracy approximations for the gradients $\partial \psi / \partial x, \partial \psi / \partial y$ can be obtained from [10] and corresponding approximations for the velocities are given below. (For details of the derivation, see the cited 
reference.) The following approximations are compact and have a local truncation error of order $h^{4}$.

$$
\begin{aligned}
& u_{0}=\left(\psi_{2}-\psi_{4}\right) / 3 h+\left(\psi_{5}+\psi_{6}-\psi_{7}-\psi_{3}\right) / 12 h+h\left(\zeta_{2}-\zeta_{4}\right) / 12 \\
& v_{0}=\left(\psi_{3}-\psi_{1}\right) / 3 h-\left(\psi_{5}-\psi_{6}-\psi_{7}+\psi_{3}\right) / 12 h+h\left(\zeta_{3}-\zeta_{1}\right) / 12
\end{aligned}
$$

\section{MODEL PROBLEM}

As a model problem, we consider the steady flow of an incompressible viscous fluid in a square cavity $(0 \leq x \leq 1$, $0 \leq y \leq 1)$. The flow is induced by the sliding motion of the top wall $(y=1)$ from right to left, and is described by the Navier-Stokes equations $(5)-(7)$. The boundary conditions are those of no slip: on the stationary walls $u=0$ and $v=0$; on the sliding wall $u=-1$ and $v=0$ (see Figure 2).

A large number of investigators have used this model problem to test new schemes and solution methods, (see for example $[4,5,6,7,8,9,11,17,18,20,22]$ and references given therein). Highly accurate benchmark solutions of this problem are available in the literature. In particular, Ghia et al. [7] obtained highly accurate solutions using $256 \times 256$ grids for 100 Res10000. Schreiber and Keller [17] solved this problem using a continuation method on a sequence of grids including an $180 \times 180$ grid; Goodrich and Soh [8] used a streamfunction algorithm on a $65 \times 65 \mathrm{grid}$. These solutions facilitate comparison and assessment of new solution 
techniques. Experimental and numerical work on the threedimensional cavity has been reported by Freitas et al.[4,5]. In order to solve the driven cavity problem, we replace the Navier- Stokes equations $(5)-(6)$ by the finite difference approximations given in eq.(8)-(10). The velocities, defined in eq.(7), are calculated using either the second order approximations (11) or the fourth order compact approximations (12) in order to compare the effectiveness of these approximations. The unit square is covered by a grid of uniform mesh width $h(h=1 / N)$. The discrete approximations (8), (9) are written at each of the $(\mathrm{N}-1)^{2}$ interior grid points. Zero values are prescribed for $\psi$ on the boundary; vorticity $\zeta$ on the boundary is obtained using the Jensen formula $[11,16]$ : on the stationary walls, we define $\zeta_{0}=\left(-8 \psi_{1}+\psi_{2}\right) / 2 \mathrm{~h}^{2}$; on the sliding wall $\mathrm{y}=1$, we define $\zeta_{0}=\left(-8 \psi_{1}+\psi_{2}\right) / 2 h^{2}+3 / h$. Here the subscript ' 0 ' denotes a grid point on the boundary; the points ' 1 ', ' 2 ' lie inside the flow region such that the points $0,1,2$ all lie on the straight line normal to the boundary (see Figure 3). These approximations have local truncation error of second order. Higher order approximations could also be defined for obtaining boundary values of $\zeta$; it is anticipated that the impact on the accuracy of the computed solutions would be marginal. These and other boundary approximations for vorticity were studied in detail by Gupta and Manohar [11] (see also Gajjar[6]). 
An inner- outer iteration procedure is utilized to obtain the numerical solutions. At each outer iteration, the linear systems from the discrete streamfunction and vorticity equations are solved iteratively. We solved these linear systems using point-S.O.R. iteration with the relaxation parameters 1.7 and 1.2 , respectively. The inner iterations are allowed no more than a preset number (usually 10) of iterations. As the fourth order approximations of the vorticity equation are stable, the inner iterations are convergent for all values of Re.

We obtained numerical solutions of the driven cavity problem for Reynolds number ranging between 1 and 2000. The solutions were obtained on a $21 \times 21$ grid $(h=1 / 20)$ and a $41 \times 41$ grid $(h=1 / 40)$. All iterations were started with zero initial data and were terminated when the maximum difference between successive approximations of $\psi, \zeta$ was smaller than $10^{-4}$. The computations were carried out on an IBM 4381 at The George Washington University and on a CRAY XMP24 at NASA Lewis Research Center.

In Table 1, we present the representative parameters of the driven cavity problem for the $41 \times 41$ grid, obtained using the fourth order approximations (12) for the velocities. This table contains the values of $\psi, \zeta$ at $V C$ ( $V C=$ center of the primary vortex), the values of $\psi$ at the centers of the secondary vortices in the lower corners, and the value of $\zeta$ at the mid-point $(.5,1)$ of the moving wall. These 
parameters are the major indicators of the accuracy of the computed solutions [11] and are quoted by most authors.

\begin{tabular}{|c|c|c|c|c|c|}
\hline $\mathrm{Re}$ & $\frac{\text { Primary }}{\psi_{\text {VC }}}$ & $\frac{\text { Vortex }}{\zeta}$ & $\frac{\text { Secondary }}{\psi}$ & $\frac{\text { Vortices }}{\psi_{\text {DVC }}}$ & $\zeta(.5,1)$ \\
\hline $\begin{array}{r}1 \\
10 \\
100 \\
400 \\
1000 \\
2000\end{array}$ & $\begin{array}{l}.100027 \\
.100029 \\
.103463 \\
.112814 \\
.111492 \\
.099586\end{array}$ & $\begin{array}{l}3.33906 \\
3.35029 \\
3.28572 \\
2.30247 \\
2.02763 \\
2.24579\end{array}$ & $\begin{array}{l}-.2091(-5) * \\
-.2212(-5) \\
-.1245(-4) \\
-.6512(-3) \\
-.1833(-2) \\
-.2849(-4)\end{array}$ & $\begin{array}{l}-.2100(-5) \\
-.2011(-5) \\
-.1747(-5) \\
-.1452(-4) \\
-.1491(-3) \\
-.7597(-4)\end{array}$ & $\begin{array}{r}5.8637 \\
5.8686 \\
6.5505 \\
10.0856 \\
15.9470 \\
18.5790\end{array}$ \\
\hline * & $2091(-5)$ & $-.2091 \times 1$ & & & \\
\hline
\end{tabular}

We also computed numerical solutions using the second order approximations (11) for the velocities. The solutions for $R e=1,10$ were found to be almost identical with those obtained using the fourth order approximation for the velocities. The representative parameters for second order velocities for $\operatorname{Re} \geq 100$ are given in Table 2 .

\begin{tabular}{|c|c|c|c|c|c|}
\hline $\mathrm{Re}$ & $\frac{\text { Primary }}{\psi_{\text {VC }}}$ & $\frac{\text { Vortex }}{\zeta \text { VC }}$ & $\frac{\text { Secondary }}{\psi_{\text {UVC }}}$ & $\frac{\text { Vortices }}{\psi_{\text {DVC }}}$ & $\zeta(.5,1)$ \\
\hline $\begin{array}{r}100 \\
400 \\
1000 \\
2000\end{array}$ & $\begin{array}{l}.103263 \\
.111151 \\
.107392 \\
.088152\end{array}$ & $\begin{array}{l}3.28369 \\
2.29561 \\
2.01499 \\
2.37916\end{array}$ & $\begin{array}{l}-.1241(-4) \\
-.7004(-3) \\
-.2108(-2) \\
-.1368(-4)\end{array}$ & $\begin{array}{l}-.1742(-5) \\
-.1367(-4) \\
-.1384(-3) \\
-.7979(-4)\end{array}$ & $\begin{array}{r}6.5641 \\
10.1538 \\
16.2462 \\
20.6838\end{array}$ \\
\hline
\end{tabular}

Representative Parameters of Driven Cavity (Second Order Velocities, $41 \times 41$ mesh)

Table 2 


\section{COMPARISON WITH EXISTING SOLUTIONS}

We now present a comparison of our solutions with the high accuracy solutions available in the literature (see, for example $[7,8,17])$. Qualitatively, our solutions exhibit the well known features of the driven cavity, including the main vortex in the central part of the cavity and secondary vortices in the lower corners. Figures 4-7 present the streamfunction and vorticity contours for $\mathrm{Re}=1$ and 100 using the $O\left(h^{4}\right)$ approximation for velocities. Figures 8-11 contain the streamfunction and vorticity contours for $\operatorname{Re}=400$ using $O\left(h^{2}\right)$ and $O\left(h^{4}\right)$ approximations for velocities. Figures 12-15 contain similar contours for $\operatorname{Re}=1000$. It is apparent that our streamfunction and vorticity contours are consistent with the published data $[5,7,8,17,18]$.

Quantitatively, our solutions using the $41 \times 41$ mesh compare very well with the highly accurate benchmark solutions available in the literature. In Table 3, we compare the values of streamfunction $\psi$ at VC and the location of $V C$ with the results from $[7,8,17]$ as applied to the driven cavity configuration shown in Figure 3 . We note that the locations of the vortex center VC using the fourth order approximations for the velocities are within the cellwidth $h(h=.025)$ of the reference data.

Taking the results of Ghia et al.[7] as the benchmark solutions, we compute the relative errors of the $\psi$ values at VC for the solutions obtained by us, by Goodrich and Soh 


\begin{tabular}{|c|c|c|c|}
\hline $\operatorname{Re}$ & $\begin{array}{l}\text { Second Order } \\
\text { Velocities }\end{array}$ & $\begin{array}{l}\text { Fourth Order } \\
\text { Velocities }\end{array}$ & $\begin{array}{l}\text { Reference } \\
\text { Data }\end{array}$ \\
\hline 1 & $\begin{array}{r}.100027 \\
(.5, .775)\end{array}$ & $\begin{array}{r}.100027 \\
(.5, .775)\end{array}$ & $\begin{array}{c}.10006 \\
(.5, .7667)^{[17]}\end{array}$ \\
\hline \multirow[t]{2}{*}{100} & $\begin{array}{r}.103263 \\
(.375, .75)\end{array}$ & $\begin{array}{r}.103463 \\
(.375, .75)\end{array}$ & $\begin{array}{cc}.103423 & {[7]} \\
(.3828, .7344)\end{array}$ \\
\hline & & & .10330 \\
\hline \multirow[t]{2}{*}{400} & $\begin{array}{r}.111152 \\
(.45, .625)\end{array}$ & $\begin{array}{r}.112814 \\
(.45, .60)\end{array}$ & $\begin{array}{cc}.113909,[7] \\
(.4453, .6055)\end{array}$ \\
\hline & & & $\begin{array}{l}.11198 \\
.11297\end{array}$ \\
\hline \multirow[t]{2}{*}{1000} & $\begin{array}{r}.107392 \\
(.475, .600)\end{array}$ & $\begin{array}{r}.111492 \\
(.475, .575)\end{array}$ & $\begin{array}{rc}.117929 & {[7]} \\
(.4687, .5625)\end{array}$ \\
\hline & & & $\begin{array}{l}.11359 \\
.11603\end{array}$ \\
\hline
\end{tabular}

[8], and by Schreiber and keller [17]. This data, given in Table 4, shows that our solutions obtained using the fourth order approximations for the velocities are either comparable in accuracy or are somewhat more accurate than the other results.

The values of streamfunction $\psi$ at the center of the secondary vortex in the upstream corner UVC (in bottom left corner of the cavity, see Fig. 3) are given in Table 5. We also give the reference data from $[7,8,17]$ for comparison. The values of this parameter obtained using the fourth order velocity approximations are much more accurate than those 


$\begin{array}{rcccc}\text { Re } & \begin{array}{c}\text { Second Order } \\ \text { Velocities }\end{array} & \begin{array}{c}\text { Fourth Order } \\ \text { Velocities }\end{array} & \begin{array}{c}\text { Reference } \\ \text { Data }\end{array} \\ 100 & .15 \% & .04 \% & .12 \% & {[17]} \\ 400 & 2.4 \% & .96 \% & .82 \% & {[17]} \\ & & & 1.7 \% & {[8]} \\ 1000 & 8.9 \% & 5.4 \% & 1.6 \% & {[17]} \\ & & & 3.7 \% & {[8]}\end{array}$

Relative Error in the Value of $\psi$ at $\mathrm{VC}$

Table 4

obtained using the second order velocity approximations; at large values of $\mathrm{Re}$, the improvement becomes even more pronounced.

\begin{tabular}{|c|c|c|c|c|}
\hline $\operatorname{Re}$ & $\begin{array}{l}\text { Second Order } \\
\text { Velocities }\end{array}$ & $\begin{array}{l}\text { Fourth Order } \\
\text { Velocities }\end{array}$ & $\begin{array}{l}\text { Referenc } \\
\text { Data }\end{array}$ & \\
\hline 1 & $-.2091(-5)$ & $-.2091(-5)$ & $-.247 \quad(-5)$ & [17] \\
\hline 100 & $-.1241(-4)$ & $-.1245(-4)$ & $\begin{array}{l}-.1254(-4) \\
-.1320(-4)\end{array}$ & $\begin{array}{l}{\left[\begin{array}{l}7 \\
{[17}\end{array}\right]} \\
{\left[\begin{array}{l}7 \\
{[}\end{array}\right]}\end{array}$ \\
\hline 400 & $-.7004(-3)$ & $-.6512(-3)$ & $\begin{array}{l}-.6424(-3) \\
-.5749(-3) \\
-.6440(-3)\end{array}$ & $\begin{array}{l}{[7]} \\
{[8]} \\
{[17]}\end{array}$ \\
\hline 1000 & $-.2108(-2)$ & $-.1833(-2)$ & $\begin{array}{l}-.1751(-2) \\
-.1892(-2) \\
-.1700(-2)\end{array}$ & $\begin{array}{l}{[7]} \\
{[8]} \\
{[17]}\end{array}$ \\
\hline
\end{tabular}

In Table 6, we give the values of $\zeta$ at VC and compare with the available data. The agreement with the reference 
data is quite good even though the location of VC substantially effects the values of this parameter; smaller values of $h$ would locate VC more accurately and give even better agreement in the $\zeta$ values at VC. In Table 7, we give the values of $\zeta$ at the mid-point of the sliding wall $y=1$ and the only comparison data available in [7].

\begin{tabular}{|c|c|c|c|}
\hline $\operatorname{Re}$ & $\begin{array}{l}\text { Second Order } \\
\text { velocities }\end{array}$ & $\begin{array}{l}\text { Fourth Order } \\
\text { Velocities }\end{array}$ & $\begin{array}{l}\text { Reference } \\
\text { Data }\end{array}$ \\
\hline 1 & 3.339 & 3.339 & $3.232[17]$ \\
\hline 100 & 3.284 & 3.286 & $\begin{array}{l}3.167[7] \\
3.182[17]\end{array}$ \\
\hline 400 & 2.296 & 2.302 & $\begin{array}{ll}2.295 & {[7]} \\
2.281 & {[17]}\end{array}$ \\
\hline 1000 & 2.015 & 2.028 & $\begin{array}{ll}2.050 & {[7]} \\
2.026 & {[17]}\end{array}$ \\
\hline
\end{tabular}

\begin{tabular}{rccc} 
Re & $\begin{array}{c}\text { Second Order } \\
\text { Velocities }\end{array}$ & $\begin{array}{c}\text { Fourth Order } \\
\text { Velocities }\end{array}$ & $\begin{array}{c}\text { Reference } \\
\text { Data }\end{array}$ \\
1 & 5.8637 & 5.8637 & - \\
100 & 6.5641 & 6.5505 & $6.5745[7]$ \\
400 & 10.1538 & 10.0856 & $10.0545[7]$ \\
1000 & 16.2462 & 15.9470 & $14.8901[7]$ \\
\hline Value of 5 at $(.5,1)$ & \\
Table 7 &
\end{tabular}

In Table 8 , we give the extreme values of the horizontal velocity $u$ at the centreline $x=0.5$ near the bottom wall $y=0$. 
Data from [7] and relative deviation of our results from this data is given for $\mathrm{Re} \geq 100$; also given is the relative error data from [8] for $R e=400$ and 1000. Similar data is given in Table 9 for the extreme values of the vertical velocity $v$ at the centreline $y=0.5$ near the left wall $x=0$. We observe that our extreme $u$ values (Table 8) are comparable to those of [8] although our extreme $v$ values (Table 9) are somewhat more erroneous than those of [8].

\begin{tabular}{|c|c|c|c|}
\hline $\operatorname{Re}$ & $\begin{array}{l}\text { Second Order } \\
\text { velocities }\end{array}$ & $\begin{array}{l}\text { Fourth Order } \\
\text { Velocities }\end{array}$ & $\begin{array}{l}\text { Reference } \\
\text { Data }\end{array}$ \\
\hline 1 & .2065 & .2070 & - \\
\hline 100 & $\begin{array}{l}.2212 \\
(4.9 \%)\end{array}$ & $\begin{array}{l}.2223 \\
(5.4 q)\end{array}$ & .2109 \\
\hline 400 & $\begin{array}{l}.3235 \\
(1.2 \%)\end{array}$ & $\begin{array}{l}.3288 \\
(.46 \%)\end{array}$ & $\begin{array}{l}.3273 \\
(2.28)\end{array}$ \\
\hline 1000 & $\begin{array}{l}.3473 \\
(9.3 \%\end{array}$ & $\begin{array}{l}.3596 \\
(6.18)\end{array}$ & $\begin{array}{l}.3829 \\
(4.68)\end{array}$ \\
\hline
\end{tabular}

In Table 10, we compare the values of $\psi$ and $\zeta$ at vC obtained with the $21 \times 21$ and $41 \times 41$ grids and note that the values of these parameters rapidly approach towards the benchmark values when the grid is refined. The rate of convergence is somewhat slower when $R e$ is large. It is expected that on further grid refinement, the solutions for large Reynolds numbers would also exhibit rapid convergence. 


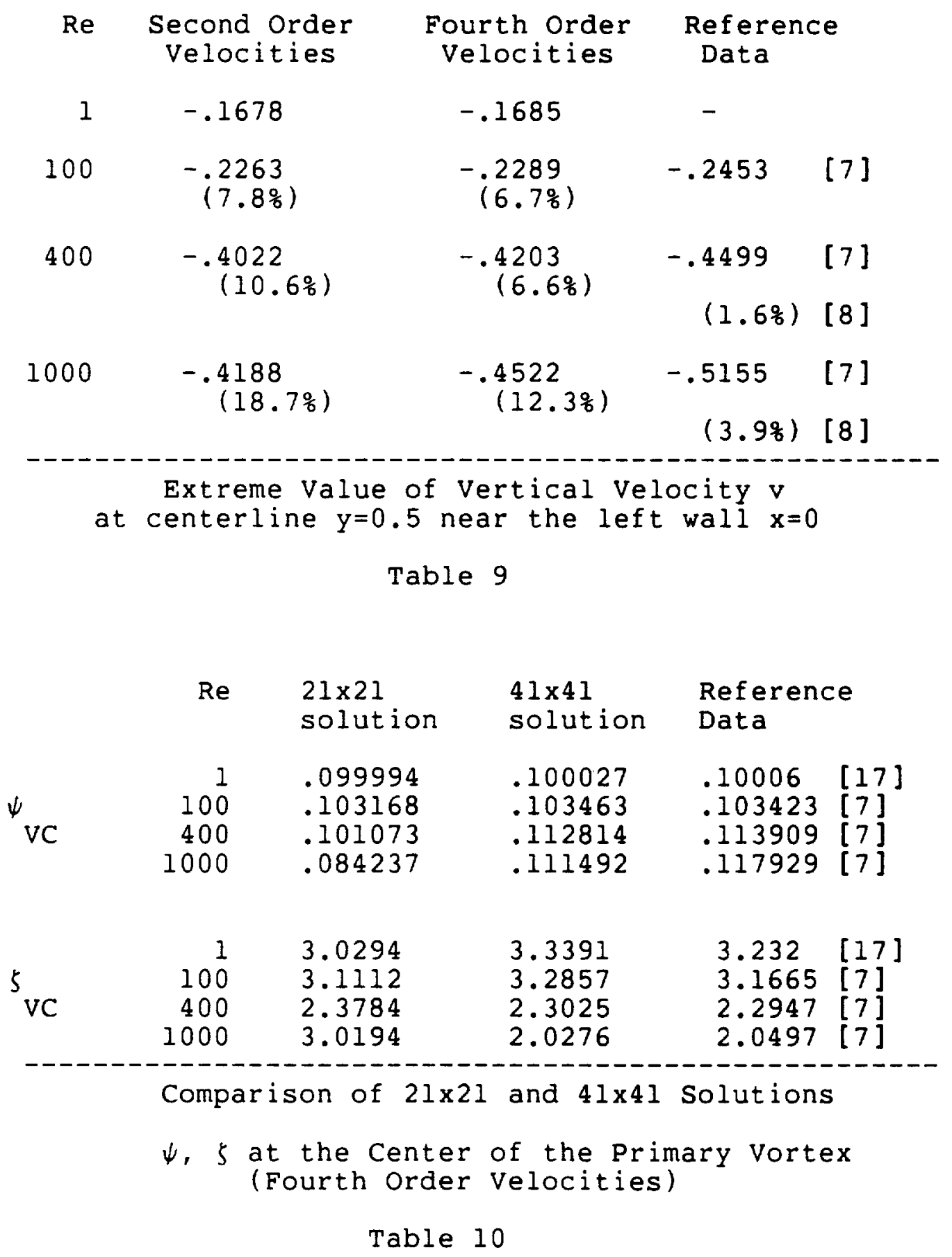




\section{DISCUSSION}

We note that for moderate values of the Reynolds number Re, the numerical solutions obtained using our high order compact difference approximations are highly accurate and compare well with the benchmark solutions available in the literature. This fact is remarkable also due to the fact that our solutions are obtained with a relatively coarse grid $(h=1 / 40)$ whereas the benchmark solutions have been obtained with fine grids (with $h$ as small as $h=1 / 256$ ).

In Table 1l, we give the number of iterations needed to converge to the required tolerance. As expected, for larger values of $\mathrm{Re}$ the convergence is slower; however, the convergence is faster with the fourth order velocity approximations than with the second order velocity approximations. We also give the CPU execution times for $\operatorname{Re}=1000,2000$ on a CRAY XMP24 in this table. With Re=1000, the convergence with fourth order velocity approximations required almost $13 \%$ less CPU time than with the second order velocity approximations; with $R e=2000$ the discrepancy increased even further. Thus, the numerical computations using the second order velocity approximations have slower convergence and produce less accurate results than the computations using the fourth order velocity approximations.

We conclude that the fourth order approximations for the Navier- Stokes equations do provide highly accurate solutions when coupled with appropriate high order 


$\begin{array}{rcc}\text { Re } & \begin{array}{c}\text { Second order } \\ \text { Velocities }\end{array} & \begin{array}{c}\text { Fourth Order } \\ \text { Velocities }\end{array} \\ 1 & 157 & 157 \\ 100 & 353 & 353 \\ 400 & 516 & 509 \\ 1000 & 1248(148 \mathrm{~s}) & 1040(127 \mathrm{~s}) \\ 2000 & >6200(>645 \mathrm{~s}) & 4266(482 \mathrm{~s})\end{array}$

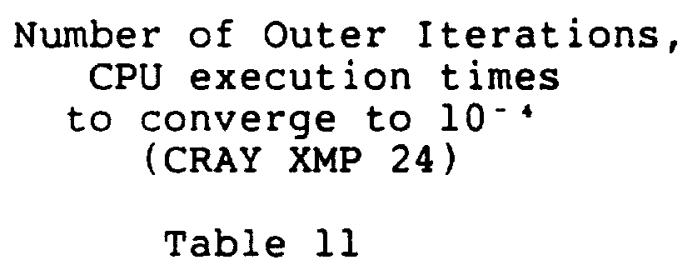

approximations for the velocities. The rate of convergence of the outer iterations slows down considerably when computing for larger values of Re. This is an on-going difficulty with all solution methods. We are currently investigating alternative methods such as the multigrid and multilevel techniques $[7,20,22]$ to obtain high accuracy driven cavity solutions for much larger values of $\mathrm{Re}$, and to solve other problems of viscous fluid flow. The results of these investigations shall be reported in the future. 


\section{REFERENCES}

1. A. Berger, J.M. Solomon, M. Ciment, S.H. Leventhal and B.C. Weinberg, Math. Comp. 35, 695 (1980).

2. G. Birkhoff and R.E. Lynch, Numerical Solution of Elliptic Equations, (SIAM, Philadelphia, 1984).

3. L. Collatz, The Numerical Treatment of Differential Equations, (Springer-Verlag, Berlin/New York, 1960).

4. C.J. Freitas, R.L. Street, A.N. Findikakis and J.R. Koseff, Internat. I. Numer. Methods Fluids 5,561 $(1985)$.

5. C.J. Freitas and R.L. Street, Internat. J. Numer. Methods Fluids 8,769 (1988).

6. J.S.B. Gajjar, Computer Phys. Comm. 37, 303 (1985).

7. U. Ghia, K.N. Ghia and C.T. Shin, J. Comput. Phys. 48, $387(1982)$.

8. J.W. Goodrich and W.Y. Soh, J. Comput. Phys. 84, 207 (1989).

9. G. Guj and F. Stella, Internat. J. Numer. Methods Fluids 8,405 (1988).

10. M.M. Gupta, J. Comput. Phys. 55, 166 (1984).

11. M.M. Gupta and R. Manohar, J. Comput. Phys. 31, 265 (1979).

12. M.M. Gupta, R.Manohar and J.W. Stephenson, Internat. J. Numer. Methods Fluids $\underline{4}, 641$ (1984).

13. M.M. Gupta, R. Manohar and J.W. Stephenson, Numerical Methods Partial Diff. Equations 1, 71 (1985).

14. R.E. Lynch and J.R. Rice, Proc. Nat. Acad. Sci. 25 , 2541 (1978).

15. R. Manohar and J.W. Stephenson, SIAM J. Sci. Stat. Comp. $\underline{5}, 69(1984)$.

16. P.J. Roache, Computational Fluid Dynamics, (Hermosa, Albuquerque, 1973). 
17. R. Schreiber and H.B. Keller, J. Comput. Phys. $\underline{49}, 310$ (1983).

18. W.A. Shay, Computers and Fluids 9, 279 (1981).

19. J.W. Stephenson, J. Comput. Phys. 55, 65 (1984).

20. S.P. Vanka and K.P. Misegades, Internat. J. Numer. Methods Fluids 7,635 (1987).

21. S.C.R. Dennis and J.D. Hudson, J. Comput. Phys. 85, 390 (1989).

22. M.C. Thompson and J. Ferziger, J. Comput. Phys. 82, 94 (1989).

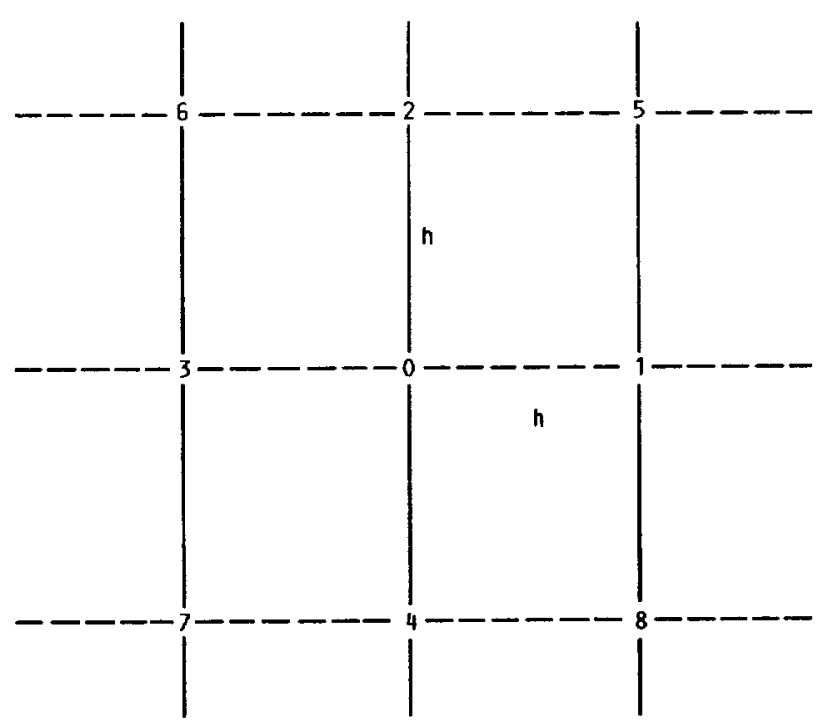

FIGURE 1. - COMPUTATIONAL STENCIL.

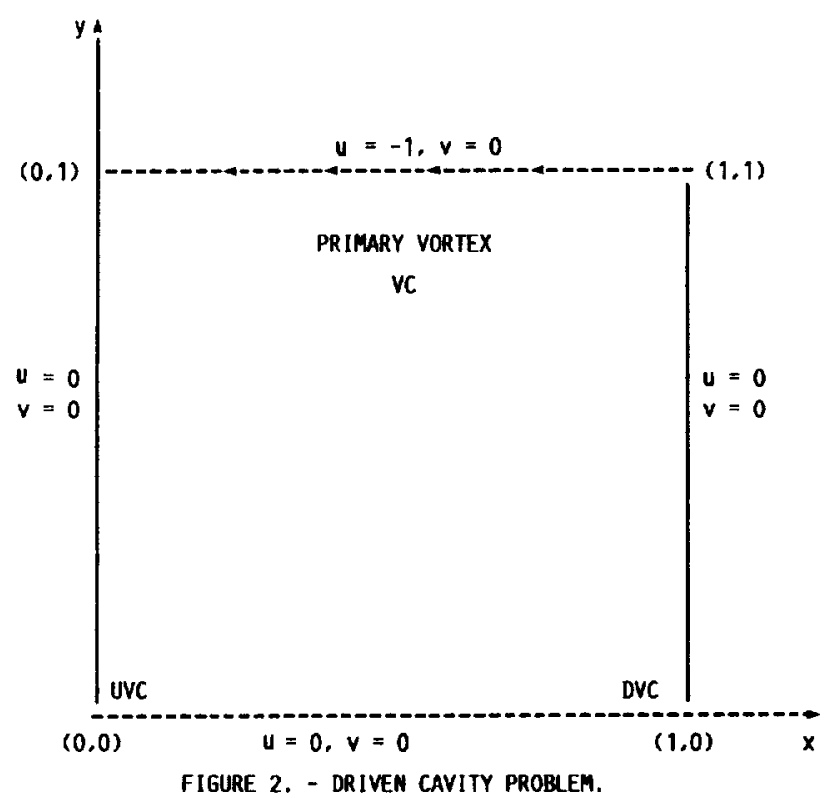

FIGURE 2. - DRIVEN CAVITY PROBLEM.

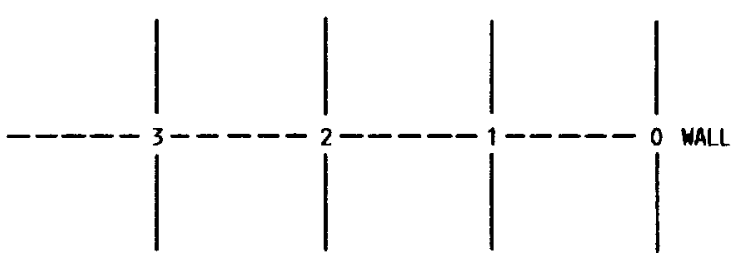

FIGURE 3. - COMPUTATIONAL STENCIL FOR WALL VORTICITY. 


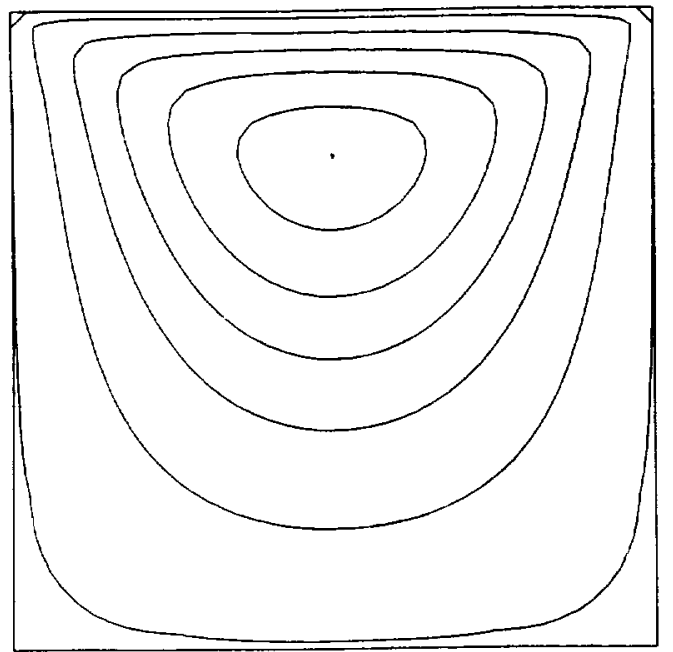

FIGURE 4. - STREAMLINES FOR Re $=1$ (FOURTH ORDER VELOC1TY).

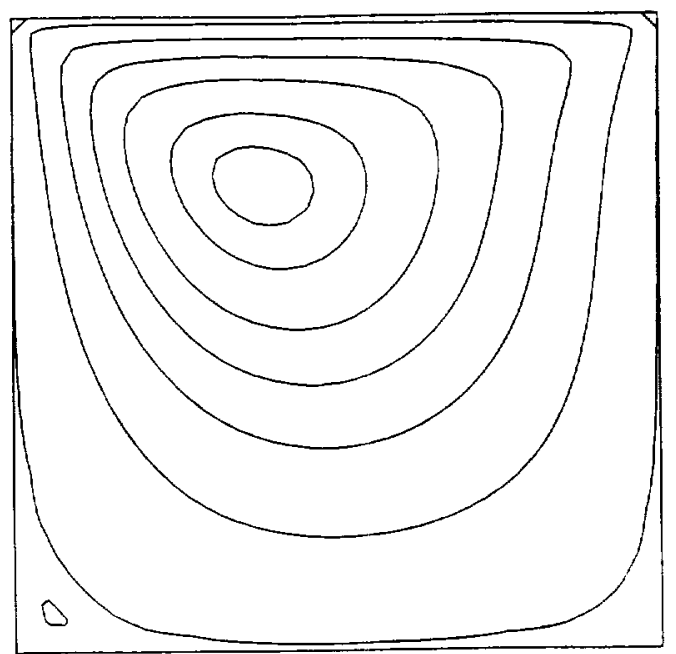

FIGURE 6 . - STREAML INES FOR Re $=100$ (FOURTH ORDER VELOCITY).

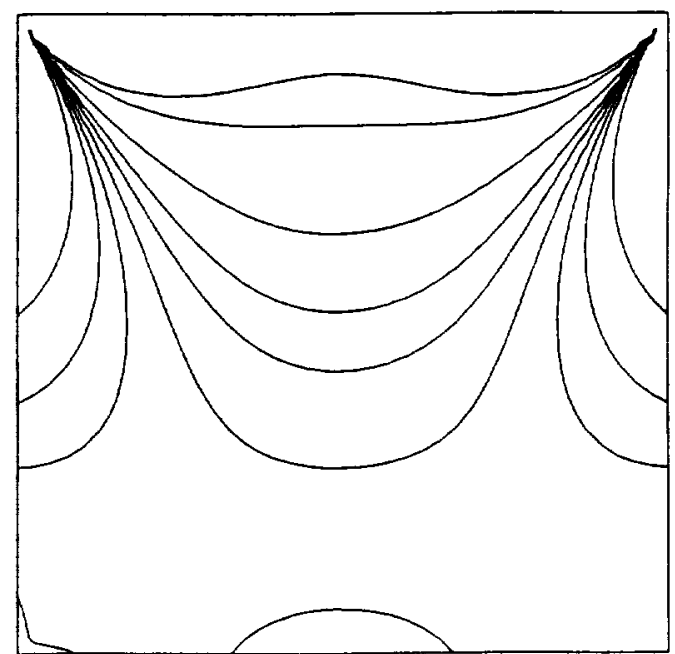

FIGURE 5. - EOUIVORTICITY CURVES FOR Re $=1$ (FOURTH ORDER VELOCITY).

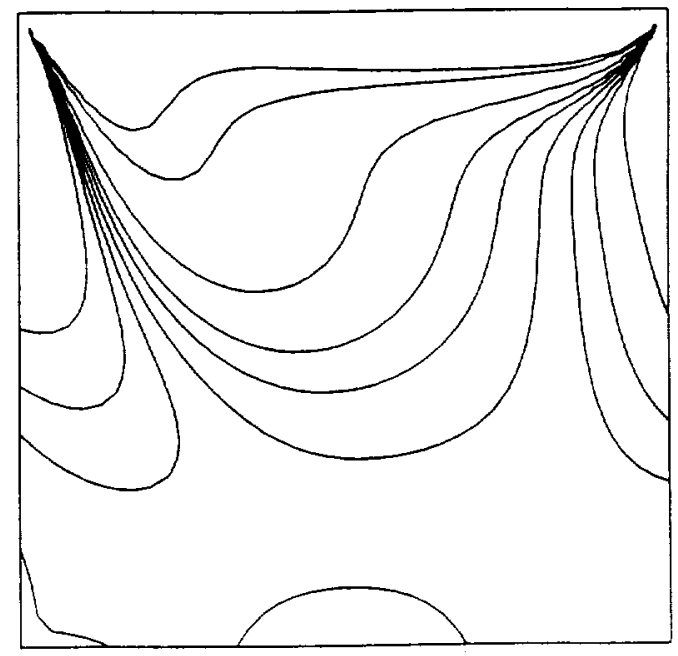

FIGURE 7. - EQUIVORTICITY CURVES FOR Re $=100$ (FOURTH ORDER VELOCITY). 


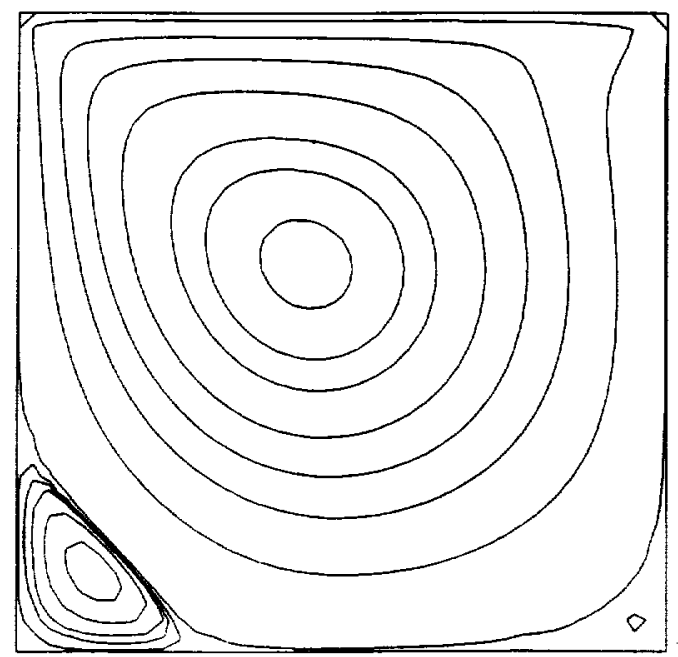

FIGURE 8. - STREAMLINES FOR Re $=400$ (FOURTH ORDER VELOCITY).

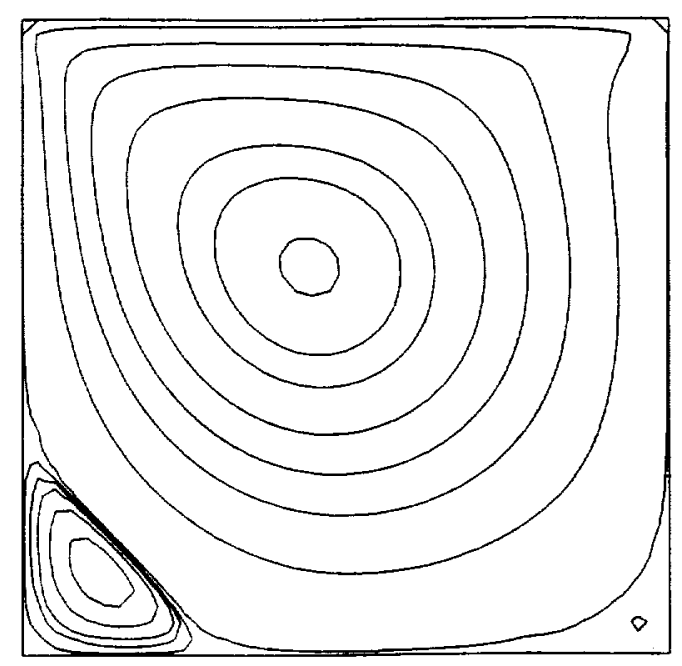

FIGURE 10. - STREAMLINES FOR Re $=400$ (SECOND ORDER VELOCITY).

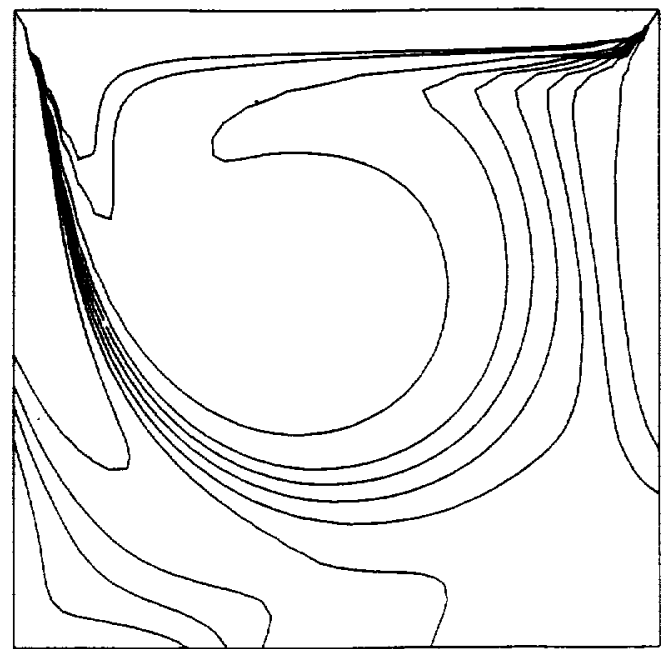

FIGURE 9. - EQUIVORTICITY CURVES FOR Re $=400$ (FOURTH ORDER VELOCITY).

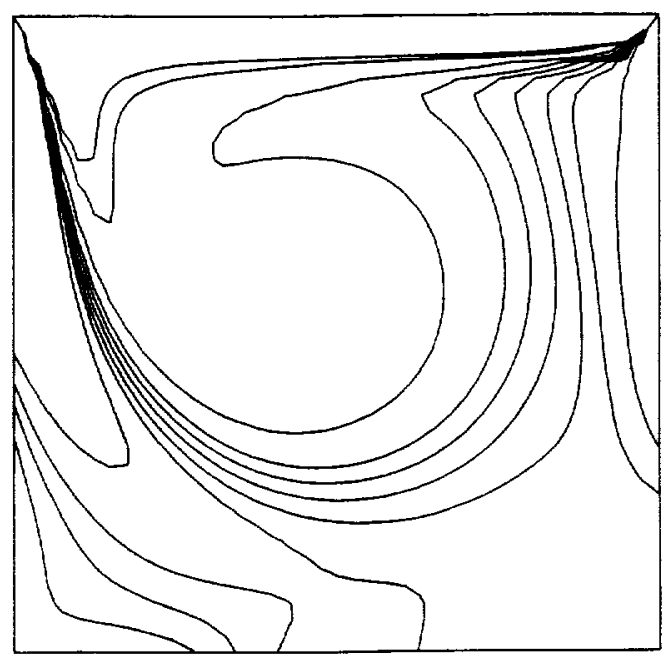

FIGURE 11. - EQUIVORTICITY CURVES FOR Re $=400$ (SECOND ORDER VELOCITY). 


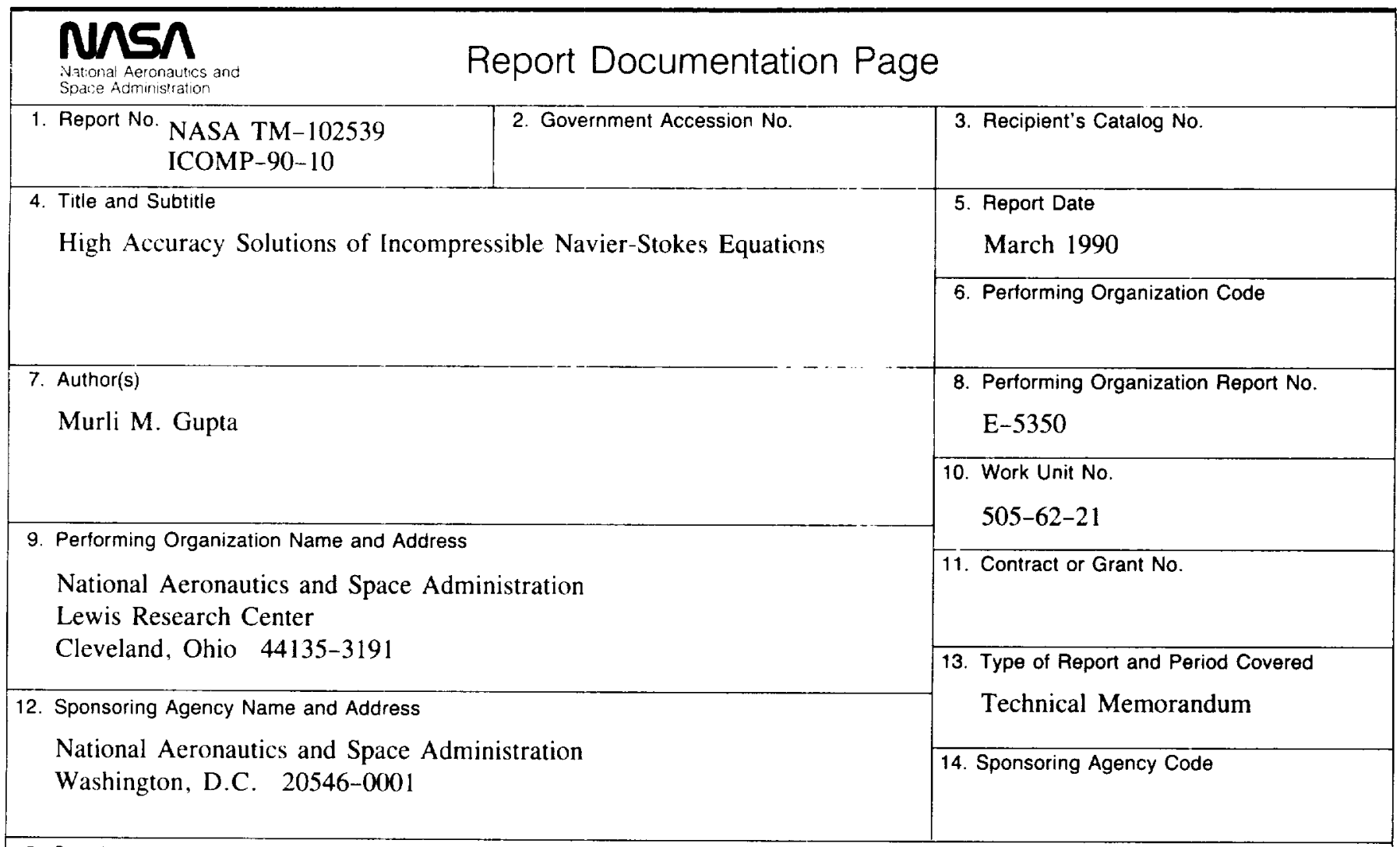

15. Supplementary Notes

Murli M. Gupta, Department of Mathematics, The George Washington University, Washington, D.C. 20052 and Institute for Computational Mechanics in Propulsion, Lewis Research Center (work funded by Space Act Agreement C-99066-G). Space Act Monitor, Louis A. Povinelli.

16. Abstract

In recent years we have developed high accuracy finite difference approximations for partial differential equations of elliptic type, with particular emphasis on the convection-diffusion equation. These approximations are of compact type, have a local truncation error of fourth order, and allow the use of standard iterative schemes to solve the resulting systems of algebraic equations. In this paper, we extend these high accuracy approximations to the solution of Navier-Stokes equations. Solutions are obtained for the model problem of driven cavity and are compared with solutions obtained using other approximations and those obtained by other authors. It is discovered that the high order approximations do indeed produce high accuracy solutions and have a potential for use in solving important problems of viscous fluid flows.

17. Key Words (Suggested by Author(s))

Navier-Stokes equations; High order approximations; Accuracy; Driven cavity problem; Viscous fluid flows
18. Distribution Statement

Unclassified-Unlimited

Subject Category 64
19. Security Classif. (of this report) Unclassified
20. Security Classif. (of this page)

Unclassified
21. No. of pages

26
22. Price* $\mathrm{A} 03$ 


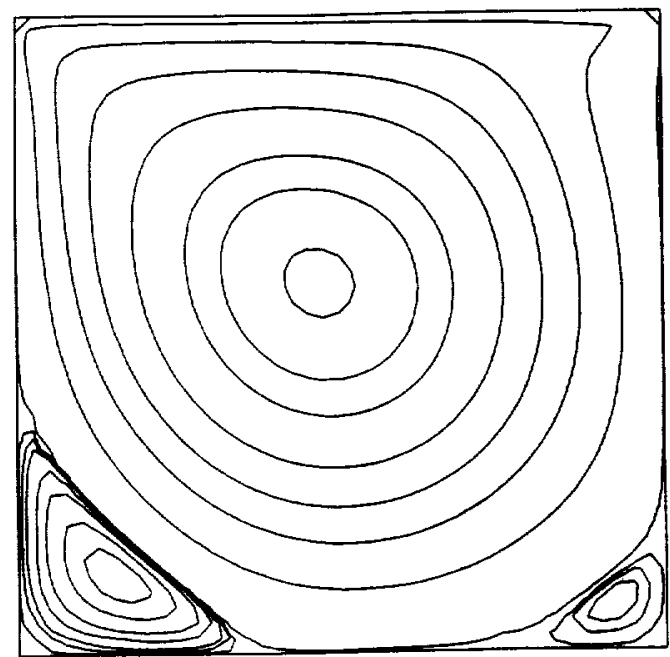

FIGURE 12. - STREAMLINES FOR Re $=1000$ (FOURTH ORDER VELOCITY).

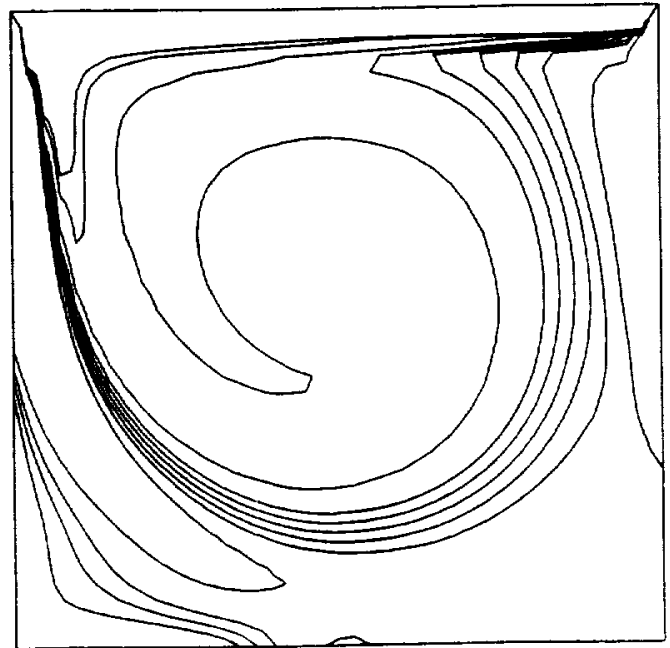

FIGURE 13. - EOUIVORTICITY CURVES FOR Re $=1000$ (FOURTH ORDER VELOCITY).

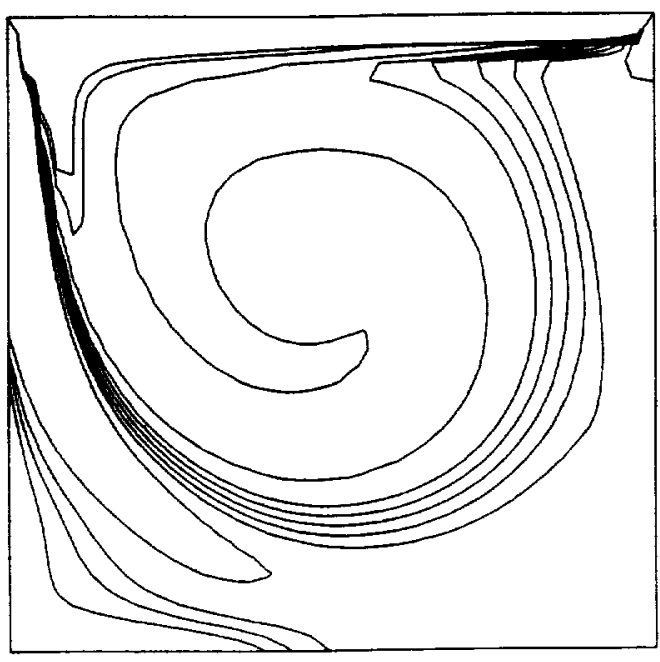

FIGURE 15. - EQUIVORTICITY CURVES FOR Re $=1000$ (SECOND ORDER VELOCITY). 


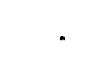


National Aeronautics and Space Administration

Lewis Research Center ICOMP (M.S. 5-3)

Cleveland, Ohio 44135

Ofliclal Business

Penalty for Privale Use $\mathbf{5 3 0 0}$
FOURTH CLASS MAIL

ADDRESS CORRECTION REQUESTED

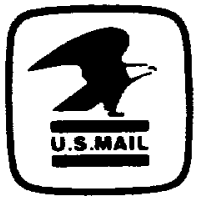

Postage and Fees Paid

Nitimat Aeronautics and

Space Adminstration

NASA 4S: 